1 Hacettepe Journal of Mathematics and Statistics

$\bigcap$ Volume 47 (1) (2018), $47-56$

\title{
On the duality of frames and fusion frames
}

Ali Akbar Arefijamaal* ${ }^{* \dagger}$, Fahimeh Arabyani Neyshaburi ${ }^{\ddagger}$ and Mitra Shamsabadi ${ }^{\S}$

\begin{abstract}
Optimal frame bounds play a key role in many applications of frame theory, such as filter banks. In this paper, we study the relation between the bounds of a frame and its alternate dual and then present some approach to construct a family of Parseval frames. Also, we survey some problems on duals of fusion frames. In particular, we discuss on some essential differences between duals of ordinary frames and fusion frames. Finally, we characterize duals of some fusion frames.
\end{abstract}

Keywords: Frame bounds; dual frames; fusion frames; dual fusion frames.

2000 AMS Classification: Primary 42C15; Secondary 41A58.

Received: 27.11.2016 Accepted : 03.05.2017 Doi : 10.15672/HJMS.2017.452

\section{Introduction and preliminaries}

Although frames were first introduced by Duffin and Schaeffer in 1952 [12], today they have been developed rapidly in mathematics and have achieved successful applications in various areas of pure and applied sciences and engineering $[2,3,4,5,6]$. Recently, due to applications and theoretical goals, some generalizations of frames have been presented $[1,7,14,18,19]$. Fusion frames are one of the most important extension of frames. In fact, fusion frames are created to model sensor networks perfectly and provide a mathematical frame work to design and analyze applications under distributed processing requirements, which share many properties with discrete frames. However, they are very different in the duality.

\footnotetext{
${ }^{*}$ Department of Mathematics and Computer Sciences, Hakim Sabzevari University, Sabzevar, Iran, Email: arefijamaal@hsu.ac.ir

${ }^{\dagger}$ Corresponding Author.

${ }^{\ddagger}$ Department of Mathematics and Computer Sciences, Hakim Sabzevari University, Sabzevar, Iran, Email: arabianif@yahoo.com

${ }^{\S}$ Department of Mathematics and Computer Sciences, Hakim Sabzevari University, Sabzevar, Iran, Email: mi.shamsabadi@hsu.ac.ir
} 
Let $\mathcal{H}$ be a separable Hilbert space and $I$ be a countable index set. A sequence $\left\{f_{i}\right\}_{i \in I} \subseteq \mathcal{H}$ is called a frame for $\mathcal{H}$ if there exist the constants $0<A \leq B<\infty$ such that

$$
A\|f\|^{2} \leq \sum_{i \in I}\left|\left\langle f, f_{i}\right\rangle\right|^{2} \leq B\|f\|^{2}, \quad(f \in \mathcal{H}) .
$$

The constants $A$ and $B$ are called frame bounds that are not unique. Supremum of all lower frame bounds is called the optimal lower frame bound and likewise, the optimal upper frame bound is defined as the infimum of all upper frame bounds. If $A=B$, we call $\left\{f_{i}\right\}_{i \in I}$ a tight frame, and in the case of $A=B=1$ we call it a Parseval frame. We say that $\left\{f_{i}\right\}_{i \in I}$ is a Bessel sequence whenever in equation of (1.1), the right-hand side holds. Given a frame $F=\left\{f_{i}\right\}_{i \in I}$, the frame operator is defined by

$$
S_{F} f=\sum_{i \in I}\left\langle f, f_{i}\right\rangle f_{i} .
$$

It is a bounded, invertible, and self-adjoint operator [11]. For a Bessel sequence $\left\{f_{i}\right\}_{i \in I}$, the synthesis operator $T_{F}: l^{2} \rightarrow \mathcal{H}$ is defined by $T_{F}\left\{c_{i}\right\}=\sum_{i \in I} c_{i} f_{i}$. If $\left\{f_{i}\right\}_{i \in I}$ is a frame, then $S_{F}=T_{F} T_{F}^{*}$ where $T_{F}^{*}: \mathcal{H} \rightarrow l^{2}$ the adjoint of $T$, given by $T_{F}^{*} f=\left\{\left\langle f, f_{i}\right\rangle\right\}_{i \in I}$, is called the analysis operator. Moreover, the optimal bounds $A$ and $B$ for a frame $F=\left\{f_{i}\right\}_{i \in I}$ of $\mathcal{H}$ are given by

$$
A=\left\|S_{F}^{-1}\right\|^{-1}=\left\|T_{F}^{\dagger}\right\|^{-2}, \quad B=\left\|S_{F}\right\|=\left\|T_{F}\right\|^{2},
$$

where $T_{F}^{\dagger}$ is the pseudo inverse of $T_{F}$, see [11].

Since the frame operator is invertible, we can reconstruct vectors in the space by

$$
f=S_{F}^{-1} S_{F} f=\sum_{i \in I}\left\langle f, S_{F}^{-1} f_{i}\right\rangle f_{i}, \quad(f \in \mathcal{H}) .
$$

The family $\left\{S_{F}^{-1} f_{i}\right\}_{i \in I}$ is also a frame for $\mathcal{H}$, so called the canonical dual frame. In general, Bessel sequence $\left\{g_{i}\right\}_{i \in I} \subseteq \mathcal{H}$ is called an alternate dual or simply a dual for Bessel sequence $\left\{f_{i}\right\}_{i \in I}$ if

$$
f=\sum_{i \in I}\left\langle f, g_{i}\right\rangle f_{i}, \quad(f \in \mathcal{H}) .
$$

There are some characterizations of dual frames [10, 11]. For example there exists a one-to-one correspondence between duals a frame $F$ and the left bounded inverses of $T_{F}^{*}$. Also, the following proposition describes a characterization of alternate dual frames.

1.1. Proposition. [2] Let $F=\left\{f_{i}\right\}_{i \in I}$ be a frame for $\mathcal{H}$ and $\left\{\delta_{i}\right\}_{i \in I}$ the standard orthonormal basis of $\ell^{2}$. Then $\left\{g_{i}\right\}_{i \in I}$ is a dual for $\left\{f_{i}\right\}_{i \in I}$ if and only if $g_{i}=S_{F}^{-1} f_{i}+\psi^{*} \delta_{i}$ for some operator $\psi \in B\left(\mathcal{H}, \ell^{2}\right)$ such that $T_{F} \psi=0$.

The structure of this paper is as follows: In Section 2, we discuss on the relation between the optimal bounds of a frame and its duals also we present some approaches for constructing of Parseval frames. Section 3 is devoted to survey some properties of dual fusion frames and characterizes duals of some fusion frames in Hilbert spaces.

\section{Dual frame bounds}

The relation between optimal bounds of a frame and its canonical dual is a well known dependency [11]. In fact, if $F=\left\{f_{i}\right\}_{i \in I}$ is a frame for $\mathcal{H}$ with the optimal bounds $A$ and $B$, respectively. The canonical dual $\left\{S^{-1} f_{i}\right\}_{i \in I}$ is a frame for $\mathcal{H}$ with the optimal bounds $1 / B$ and $1 / A$, respectively. In this section, we survey the relationship between optimal bounds of a frame and its alternate dual. 
2.1. Proposition. Let $F=\left\{f_{i}\right\}_{i \in I}$ be a tight frame with the optimal bound $A$, and let $\left\{g_{i}\right\}_{i \in I}$ be a tight dual of $\left\{f_{i}\right\}_{i \in I}$ with the optimal bound $1 / A$. Then $\left\{g_{i}\right\}_{i \in I}$ is the canonical dual of $F$.

Proof. Since $\left\{g_{i}\right\}_{i \in I}$ is a dual of $\left\{f_{i}\right\}_{i \in I}$, by the Proposition 1.1 there exists a mapping $\psi \in B\left(\mathcal{H}, l^{2}\right)$ such that $g_{i}=S_{F}^{-1} f_{i}+\psi^{*} \delta_{i}$ where $\left\{\delta_{i}\right\}_{i \in I}$ is the standard orthonormal basis of $l^{2}$. Therefore

$$
\begin{aligned}
\frac{1}{A} f & =\sum_{i \in I}\left\langle f, g_{i}\right\rangle g_{i}=\sum_{i \in I}\left\langle f, S_{F}^{-1} f_{i}+\psi^{*} \delta_{i}\right\rangle\left(S_{F}^{-1} f_{i}+\psi^{*} \delta_{i}\right) \\
& =S_{F}^{-1} \sum_{i \in I}\left\langle f, S_{F}^{-1} f_{i}\right\rangle f_{i}+\sum_{i \in I}\left\langle f, \psi^{*} \delta_{i}\right\rangle S_{F}^{-1} f_{i} \\
& +\sum_{i \in I}\left\langle f, S_{F}^{-1} f_{i}\right\rangle \psi^{*} \delta_{i}+\sum_{i \in I}\left\langle f, \psi^{*} \delta_{i}\right\rangle \psi^{*} \delta_{i} \\
& =S_{F}^{-1} f+\psi^{*} \psi f=\frac{1}{A} f+\psi^{*} \psi f .
\end{aligned}
$$

It implies that $\psi^{*} \psi=0$, i.e $\psi=0$ and so $\left\{g_{i}\right\}_{i \in I}$ is the canonical dual of $F$.

The following example shows that the condition tightness cannot be removed in the above proposition.

2.2. Example. Let $\left\{e_{i}\right\}_{i \in I}$ be an orthonormal basis of Hilbert space $\mathcal{H}$, and also let $\left\{f_{i}\right\}_{i \in I}=\left\{e_{1}, e_{1}, e_{2}, e_{2}, e_{3}, e_{4}, \ldots\right\}$. Then $\left\{f_{i}\right\}_{i \in I}$ is a frame for $\mathcal{H}$ with the optimal bounds 1 and 2 , respectively. Its canonical dual is

$$
\left\{S_{F}^{-1} f_{i}\right\}_{i \in I}=\left\{1 / 2 e_{1}, 1 / 2 e_{1}, 1 / 2 e_{2}, 1 / 2 e_{2}, e_{3}, e_{4}, \ldots\right\},
$$

with the optimal bounds $1 / 2$ and 1 , respectively. Now, it is not difficult to check that $\left\{g_{i}\right\}_{i \in I}=\left\{1 / 2 e_{1}, 1 / 2 e_{1}, 0, e_{2}, e_{3}, \ldots\right\}$ is also an alternate dual frame of $\left\{f_{i}\right\}_{i \in I}$ with the same optimal bounds.

Now, we are going to distinct a relation between the bounds of a frame and its alternate dual, to this end, first we recall the following definition.

2.3. Definition. Suppose that $X$ and $Y$ are Banach spaces and $T \in B(X, Y)$, the minimum modulus of $T$ is defined as the following

$$
\gamma(T)=\inf \{\|T f\|: \quad f \in X, \quad\|f\|=1\} .
$$

It is well known that $\gamma(T)>0$ if and only if $T$ is injective and has closed range, see for instance [16].

2.4. Theorem. Let $F=\left\{f_{i}\right\}_{i \in I}$ be a frame with the optimal bounds $A$ and $B$. If $G=\left\{g_{i}\right\}_{i \in I}$ is a dual of $F$ with the optimal bounds $C$ and $D$ then

$$
\begin{aligned}
& \frac{1}{B}+(\gamma(\psi))^{2} \leq C \leq\left(\|\psi\|+\frac{1}{\sqrt{B}}\right)^{2}, \\
& \frac{1}{A} \leq D \leq \frac{1}{A}+\|\psi\|^{2},
\end{aligned}
$$

where $\psi \in B\left(\mathcal{H}, l^{2}\right)$ is associated with $G$ given by Proposition 1.1.

Proof. First, we show the lower bound in 2.1,

$$
\begin{aligned}
C & =i n f_{\|f\|=1}\left\langle S_{G} f, f\right\rangle=i n f_{\|f\|=1}\left\langle S_{F}^{-1} f, f\right\rangle+\|\psi f\|^{2} \\
& \geq i n f_{\|f\|=1}\left\langle S_{F}^{-1} f, f\right\rangle+i n f_{\|f\|=1}\|\psi f\|^{2} \\
& =\frac{1}{B}+(\gamma(\psi))^{2}
\end{aligned}
$$


Also, for each $f \in \mathcal{H}$,

$$
\begin{aligned}
\sum_{i \in I}\left|\left\langle f, g_{i}\right\rangle\right|^{2} & =\sum_{i \in I}\left|\left\langle f, S_{F}^{-1} f_{i}+\psi^{*} \delta_{i}\right\rangle\right|^{2} \\
& \leq \sum_{i \in I}\left|\left\langle f, S_{F}^{-1} f_{i}\right\rangle\right|^{2}+\sum_{i \in I}\left|\left\langle f, \psi^{*} \delta_{i}\right\rangle\right|^{2}+2 \sum_{i \in I}\left|\left\langle f, S_{F}^{-1} f_{i}\right\rangle \|\left\langle f, \psi^{*} \delta_{i}\right\rangle\right| \\
& \leq \sum_{i \in I}\left|\left\langle f, S_{F}^{-1} f_{i}\right\rangle\right|^{2}+\|\psi f\|^{2}+2\|\psi f\|\left(\sum_{i \in I}\left|\left\langle f, S_{F}^{-1} f_{i}\right\rangle\right|^{2}\right)^{1 / 2} .
\end{aligned}
$$

Hence

$$
C=i n f_{\|f\| \leq 1} \sum_{i \in I}\left|\left\langle f, g_{i}\right\rangle\right|^{2} \leq\left(\|\psi\|+\frac{1}{\sqrt{B}}\right)^{2} .
$$

Moreover, for every dual frame $G=\left\{g_{i}\right\}_{i \in I}$ of $F$ by Lemma 5.3 .6 of [11] we have

$$
\sum_{i \in I}\left|\left\langle f, S_{F}^{-1} f_{i}\right\rangle\right|^{2} \leq \sum_{i \in I}\left|\left\langle f, g_{i}\right\rangle\right|^{2}
$$

so applying the fact that the canonical dual $\left\{S_{F}^{-1} f_{i}\right\}_{i \in I}$ has optimal bounds $\frac{1}{B}$ and $\frac{1}{A}$, we obtain the lower bound in (2.2). Finally, for computing of the upper bound of $D$, by using of Proposition 1.1 there exists a bounded operator $\psi$ such that $T_{F} \psi=0$ and $g_{i}=S_{F}^{-1} f_{i}+\psi^{*} \delta_{i}$ for each $i \in I$. Therefore

$$
\begin{aligned}
\left\langle S_{G} f, f\right\rangle & =\left\langle\sum_{i \in I}\left\langle f, S_{F}^{-1} f_{i}+\psi^{*} \delta_{i}\right\rangle\left(S_{F}^{-1} f_{i}+\psi^{*} \delta_{i}\right), f\right\rangle \\
& =\left\langle S_{F}^{-1} \sum_{i \in I}\left\langle S_{F}^{-1} f, f_{i}\right\rangle f_{i}, f\right\rangle+\left\langle\psi^{*} \psi f, f\right\rangle \\
& =\left\langle S_{F}^{-1} f, f\right\rangle+\|\psi f\|^{2},
\end{aligned}
$$

for each $f \in \mathcal{H}$. Now, we obtain

$$
\begin{aligned}
D & =\left\|S_{G}\right\| \\
& =\sup _{\|f\| \leq 1}\left|\left\langle S_{G} f, f\right\rangle\right| \\
& =\sup _{\|f\| \leq 1}\left|\left\langle S_{F}^{-1} f, f\right\rangle+\|\psi f\|^{2}\right| \\
& \leq \frac{1}{A}+\|\psi\|^{2} .
\end{aligned}
$$

\subsection{Remark.}

(i) However the operator $\psi$ in Theorem 2.4 may not be injective in general, there are dual frames for which the corresponding operator $\psi$ is injective and has closed range. For example, consider $\mathcal{H}=\mathbb{R}^{3}$ with the standard orthonormal basis $\left\{e_{i}\right\}_{i=1}^{3}$, also let $F=\left\{e_{1}, e_{1}, \frac{1}{\sqrt{2}} e_{2}, \frac{1}{\sqrt{2}} e_{2}, \frac{1}{\sqrt{3}} e_{3}, \frac{1}{\sqrt{3}} e_{3}, \frac{1}{\sqrt{3}} e_{3}\right\}$. Then $F$ is a frame of $\mathcal{H}$, with the canonical dual

$$
S_{F}^{-1} F=\left\{\frac{1}{2} e_{1}, \frac{1}{2} e_{1}, \frac{1}{\sqrt{2}} e_{2}, \frac{1}{\sqrt{2}} e_{2}, \frac{1}{\sqrt{3}} e_{3}, \frac{1}{\sqrt{3}} e_{3}, \frac{1}{\sqrt{3}} e_{3}\right\}
$$

On the other hand, the sequense $G=\left\{e_{1}, 0, \sqrt{2} e_{2}, 0, \sqrt{3} e_{3}, 0,0\right\}$ is a dual frame of $F$, and we have

$$
G-S_{F}^{-1} F=\left\{\frac{1}{2} e_{1},-\frac{1}{2} e_{1}, \frac{1}{\sqrt{2}} e_{2},-\frac{1}{\sqrt{2}} e_{2}, \frac{2}{\sqrt{3}} e_{3},-\frac{1}{\sqrt{3}} e_{3},-\frac{1}{\sqrt{3}} e_{3}\right\} .
$$


Since $G-S_{F}^{-1} F$ is a frame for $\mathcal{H}$, so $\psi=T_{G}^{*}-T_{F}^{*} S_{F}^{-1}$ is an injective operator and has closed range, i.e., $\gamma(\psi)>0$.

(ii) There are some frames in Hilbert spaces for which there exist dual frames with arbitrary large optimal bounds. For example, let $\left\{e_{i}\right\}_{i \in I}$ be an orthonormal basis of $\mathcal{H}$. Then $F=\left\{e_{1}, 0, e_{2}, 0, e_{3}, 0, \ldots\right\}$ is a Parseval frame for $\mathcal{H}$ and the sequence $G=\left\{e_{1}, n e_{1}, e_{2}, n e_{2}, e_{3}, n e_{3}, \ldots\right\}$ is a dual of $F$, for each $n \in \mathbb{N}$. Moreover,

$$
\sum_{i \in I}\left|\left\langle f, g_{i}\right\rangle\right|^{2}=\left(1+n^{2}\right)\|f\|^{2} \rightarrow \infty, \quad(n \rightarrow \infty) .
$$

The following proposition, for a frame $F=\left\{f_{i}\right\}_{i \in I}$, characterizes all operators $U \in$ $B(\mathcal{H})$, where $\left\{U f_{i}\right\}_{i \in I}$ is a Parseval frame. As a particular case, when $U=S_{F}^{-1 / 2}$ we obtain the well-known Parseval frame $\left\{S_{F}^{-1 / 2} f_{i}\right\}_{i \in I}$. The proof is obtained by a straightforward calculation.

2.6. Proposition. Let $\left\{f_{i}\right\}_{i \in I}$ be a frame for $\mathcal{H}$ with the operator frame $S$, and $U \in$ $B(\mathcal{H})$. Then $\left\{U f_{i}\right\}_{i \in I}$ is a Parseval frame if and only if $U S_{F} U^{*}=I_{\mathcal{H}}$.

In the end of this section we are going to construct a family of Parseval frames from an alternate dual. In fact let $\left\{g_{i}\right\}_{i \in I}$ be a dual frame of $\left\{f_{i}\right\}_{i \in I}$. Take

$$
\psi=T_{G}^{*}-T_{F}^{*} S_{F}^{-1} .
$$

Then

$$
S_{G}=S_{F}^{-1}+\psi^{*} \psi \text {. }
$$

Hence $\left\{\left(S_{F}^{-1}+\psi^{*} \psi\right)^{-1 / 2} g_{i}\right\}_{i \in I}$ is a Parseval frame. By replacing $\psi$ with $\psi S_{F}$, we can obtain the following family of Parseval frames as,

$$
\left\{\left(S_{F}^{-1}+S_{F}^{n} \psi^{*} \psi S_{F}^{n}\right)^{-1 / 2}\left(S_{F}^{-1} f_{i}+S_{F}^{n} \psi^{*} \delta_{i}\right)\right\}_{i \in I}, \quad(n \in \mathbb{N})
$$

\section{Fusion frames}

In this section, we first briefly recall the basic definitions and notations of fusion frames. Then we give some results on dual fusion frames. Throughout this section, $\pi_{V}$ denotes the orthogonal projection from $\mathcal{H}$ onto a closed subspace $V$.

3.1. Definition. Let $\left\{W_{i}\right\}_{i \in I}$ be a family of closed subspaces of $\mathcal{H}$ and $\left\{\omega_{i}\right\}_{i \in I}$ be a family of weights, i.e. $\omega_{i}>0, i \in I$. Then $\left\{\left(W_{i}, \omega_{i}\right)\right\}_{i \in I}$ is called a fusion frame for $\mathcal{H}$ if there exist the constants $0<A \leq B<\infty$ such that

$$
A\|f\|^{2} \leq \sum_{i \in I} \omega_{i}^{2}\left\|\pi_{W_{i}} f\right\|^{2} \leq B\|f\|^{2}, \quad(f \in \mathcal{H})
$$

The constants $A$ and $B$ are called fusion frame bounds. If we only have the upper bound in (3.1) we call $\left\{\left(W_{i}, \omega_{i}\right)\right\}_{i \in I}$, a Bessel fusion sequence. A fusion frame is called tight, if $A=B$, and Parseval if $A=B=1$. Also if $\omega_{i}=\omega$ for all $i \in I$, the collection $\left\{\left(W_{i}, \omega_{i}\right)\right\}_{i \in I}$ is called $\omega$-uniform. A fusion frame $\left\{\left(W_{i}, \omega_{i}\right)\right\}_{i \in I}$ is said to be an orthonormal fusion basis if $\mathcal{H}=\bigoplus_{i \in I} W_{i}$. Recall that for each sequence $\left\{W_{i}\right\}_{i \in I}$ of closed subspaces in $\mathcal{H}$, the space

$$
\sum_{i \in I} \oplus W_{i}=\left\{\left\{f_{i}\right\}_{i \in I}: f_{i} \in W_{i}, \sum_{i \in I}\left\|f_{i}\right\|^{2}<\infty\right\},
$$

with the inner product $\left\langle\left\{f_{i}\right\}_{i \in I},\left\{g_{i}\right\}_{i \in I}\right\rangle=\sum_{i \in I}\left\langle f_{i}, g_{i}\right\rangle$ is a Hilbert space. For a Bessel fusion sequence $\left\{\left(W_{i}, \omega_{i}\right)\right\}_{i \in I}$ of $\mathcal{H}$, the synthesis operator $T_{W}: \sum_{i \in I} \oplus W_{i} \rightarrow \mathcal{H}$ is 
defined by

$$
T_{W}\left(\left\{f_{i}\right\}_{i \in I}\right)=\sum_{i \in I} \omega_{i} f_{i}, \quad\left(\left\{f_{i}\right\} \in \sum_{i \in I} \oplus W_{i}\right) .
$$

Its adjoint operator $T_{W}^{*}: \mathcal{H} \rightarrow \sum_{i \in I} \oplus W_{i}$, which is called the analysis operator, is obtained by $T_{W}^{*} f=\left\{\omega_{i} \pi_{W_{i}} f\right\}_{i \in I}$. If $\left\{\left(W_{i}, \omega_{i}\right)\right\}_{i \in I}$ is a fusion frame, the fusion frame operator $S_{W}: \mathcal{H} \rightarrow \mathcal{H}$ defined by $S_{W} f=T_{W} T_{W}^{*} f=\sum_{i \in I} \omega_{i}^{2} \pi_{W_{i}} f$ is a bounded, invertible as well as positive. A connection between local and global properties is given in the next result, see [7].

3.2. Theorem. For each $i \in I$, let $W_{i}$ be a closed subspace of $\mathcal{H}$ and $\omega_{i}>0$. Also let $\left\{f_{i, j}\right\}_{j \in J_{i}}$ be a frame for $W_{i}$ with frame bounds $\alpha_{i}$ and $\beta_{i}$ such that

$$
0<\alpha=i n f_{i \in I} \alpha_{i} \leq \beta=\sup _{i \in I} \beta_{i}<\infty .
$$

Then the following conditions are equivalent.

(i) $\left\{\left(W_{i}, \omega_{i}\right)\right\}_{i \in I}$ is a fusion frame of $\mathcal{H}$ with bounds $C$ and $D$.

(ii) $\left\{\omega_{i} f_{i, j}\right\}_{i \in I, j \in J_{i}}$ is a frame of $\mathcal{H}$ with bounds $\alpha C$ and $\beta D$, .

For every fusion frame as $\left\{\left(W_{i}, \omega_{i}\right)\right\}_{i \in I}$, there exist frames $\left\{f_{i, j}\right\}_{j \in J_{i}}$ for $W_{i}$, such that satisfy (3.2), [7]. These frames are called the local frames of $\left\{\left(W_{i}, \omega_{i}\right)\right\}_{i \in I}$.

A Bessel fusion sequence $\left\{\left(V_{i}, \nu_{i}\right)\right\}_{i \in I}$ is called a dual of $\left\{\left(W_{i}, \omega_{i}\right)\right\}_{i \in I}$ if [13]

$$
f=\sum_{i \in I} \omega_{i} \nu_{i} \pi_{V_{i}} S_{W}^{-1} \pi_{W_{i}} f, \quad(f \in \mathcal{H})
$$

Every fusion frame $W=\left\{\left(W_{i}, \omega_{i}\right)\right\}_{i \in I}$ has a dual as $\left\{\left(S_{W}^{-1} W_{i}, \omega_{i}\right)\right\}_{i \in I}$, so called the canonical dual. Also, it is not difficult to see that a Bessel fusion sequence $\left\{\left(V_{i}, v_{i}\right)\right\}_{i \in I}$ is a dual of fusion frame $\left\{\left(W_{i}, \omega_{i}\right)\right\}_{i \in I}$ if and only if $T_{V} \phi_{v w} T_{W}^{*}=I_{\mathcal{H}}$, where the bounded operator $\phi_{v w}: \sum_{i \in I} \bigoplus W_{i} \rightarrow \sum_{i \in I} \bigoplus V_{i}$ is given by

$$
\phi_{v w}\left(\left\{f_{i}\right\}_{i \in I}\right)=\left\{\pi_{V_{i}} S_{W}^{-1} f_{i}\right\}_{i \in I} .
$$

In [15], it is proved that for a fusion frame $W=\left\{\left(W_{i}, \omega_{i}\right)\right\}_{i \in I}$ with the frame operator $S_{W}$, a Bessel fusion sequence $V=\left\{\left(V_{i}, \omega_{i}\right)\right\}_{i \in I}$ that $V_{i}=S_{W}^{-1} W_{i} \oplus U_{i}$, is dual of $W$ in which $U_{i}$ is a closed subspace of $\mathcal{H}$ for all $i \in I$. Although, there is no complete characterization of dual fusion frames.

3.1. Opposite relations between duals of frames and fusion frames. In the sequel, we present some discussions on duals of fusion frames. In particular, we show that unlike ordinary frames, there is no one-to-one correspondence between duals of a fusion frame and the bounded left inverses of its analysis operator. In the following proposition, we investigate a relation between dual fusion frames and some their local frames.

3.3. Proposition. Let $W=\left\{\left(W_{i}, \omega_{i}\right)\right\}_{i \in I}$ be a fusion frame for $\mathcal{H}$ and $\left\{e_{j}\right\}_{j \in J}$ be an orthonormal basis for $\mathcal{H}$. Then a Bessel sequence $V=\left\{\left(V_{i}, v_{i}\right)\right\}_{i \in I}$ is a dual of $W$ if and only if $G=\left\{v_{i} \pi_{V_{i}} e_{j}\right\}_{i \in I, j \in J}$ is a dual frame of $F=\left\{\omega_{i} \pi_{W_{i}} S_{W}^{-1} e_{j}\right\}_{i \in I, j \in J}$. In particular, $W$ is also a dual of $V$ if $S_{W}=S_{V}$.

Proof. The result is obtained from the following equalities

$$
\begin{aligned}
\sum_{i \in I, j \in J}\left\langle f, \omega_{i} \pi_{W_{i}} S_{W}^{-1} e_{j}\right\rangle v_{i} \pi_{V_{i}} e_{j} & =\sum_{i \in I, j \in J} \omega_{i} v_{i} \pi_{V_{i}}\left\langle S_{W}^{-1} \pi_{W_{i}} f, e_{j}\right\rangle e_{j} \\
& =\sum_{i \in I} \omega_{i} v_{i} \pi_{V_{i}} S_{W}^{-1} \pi_{W_{i}} f
\end{aligned}
$$


Moreover, since $F$ is also a dual of $G$, we have

$$
\begin{aligned}
f & =\sum_{i \in I, j \in J}\left\langle f, v_{i} \pi_{V_{i}} e_{j}\right\rangle \omega_{i} \pi_{W_{i}} S_{W}^{-1} e_{j} \\
& =\sum_{i \in I, j \in J} \omega_{i} v_{i} \pi_{W_{i}} S_{W}^{-1}\left\langle\pi_{V_{i}} f, e_{j}\right\rangle e_{j} \\
& =\sum_{i \in I} \omega_{i} v_{i} \pi_{W_{i}} S_{V}^{-1} \pi_{V_{i}} f=T_{W} \varphi_{w, v} T_{V}^{*} f,
\end{aligned}
$$

for each $f \in \mathcal{H}$. This means that $W$ is also a dual of $V$.

It is worthwhile to mention that two fusion frames are not dual of each other in general. As an easy consequence of Proposition 3.3, the result holds if fusion frames are Parseval.

Now, we are going to discuss on some differences between fusion frames and ordinary frames. First, let $\left\{\left(W_{i}, \omega_{i}\right)\right\}_{i \in I}$ be a fusion frame of $\mathcal{H}$ and $T \in B(\mathcal{H})$ be an invertible operator. It is well known that $\left\{\left(T W_{i}, \omega_{i}\right)\right\}_{i \in I}$ is also a fusion frame of $\mathcal{H}$, see [8]. However, in fusion frames unlike ordinary frames a surjective operator $T \in B(\mathcal{H})$ may not preserve Besselian property. The next example shows this fact.

3.4. Example. Let $\left\{e_{i}\right\}_{n \in I}$ be an orthonormal basis of $\mathcal{H}$ and $W_{i}=\operatorname{span}\left\{e_{i}\right\}$, for all $i \in I$. Clearly $\left\{\left(W_{i}, 1\right)\right\}$ is an orthonormal fusion basis for $\mathcal{H}$. Define

$$
T e_{i}=\left\{\begin{array}{cc}
\frac{1}{m} e_{1} & i=2 m-1 \\
e_{m} & i=2 m .
\end{array}\right.
$$

Then the mapping $T$ can be extended to a bounded and surjective linear operator on $\mathcal{H}$, i.e $T \in B(\mathcal{H})$. Also

$$
T W_{i}=\left\{\begin{array}{cc}
\operatorname{span}\left\{e_{1}\right\} & i=2 m-1, \\
\operatorname{span}\left\{e_{m}\right\} & i=2 m .
\end{array}\right.
$$

Hence, we can easily see that for $f=e_{1}$

$$
\sum_{i=1}^{n}\left\|\pi_{T W_{i}} f\right\|^{2} \rightarrow \infty, \quad(n \rightarrow \infty),
$$

i.e. $\left\{\left(T W_{i}, 1\right)\right\}$ is not a Bessel fusion sequence.

See [17] for more examples. Also, the construction of Parseval fusion frames, which have a key role in fusion frame theory is different from ordinary frames. More precisely, let $\left\{\left(W_{i}, \omega_{i}\right)\right\}_{i \in I}$ be a fusion frame with bounds $A$ and $B$, we can see that $\left\{\left(S_{W}^{-1 / 2} W_{i}, \omega_{i}\right)\right\}_{i \in I}$ is not a Parseval fusion frame, in general. Moreover, there exists some fusion frames for $\mathcal{H}$ such that for every invertible operator $U \in B(\mathcal{H}),\left\{U W_{i}\right\}_{i \in I}$ fails to be a Parseval fusion frame, see Example 7.6 of [17].

Now, we show that Proposition 1.1 for fusion frames fails. Let $W=\left\{\left(W_{i}, \omega_{i}\right)\right\}_{i \in I}$ be a fusion frame with a dual as $\left\{\left(V_{i}, v_{i}\right)\right\}_{i \in I}$. Then there exists an operator $\Psi \in$ $B\left(\mathcal{H}, \sum_{i \in I} \bigoplus W_{i}\right)$ such that $T_{W} \Psi=0$. In fact there exists a bounded operator $\phi_{v w}$, given by (3.4), such that $T_{V} \phi_{v w} T_{W}^{*}=I_{\mathcal{H}}$. Take

$$
\Psi=\phi_{v w}^{*} T_{V}^{*}-T_{W}^{*} S_{W}^{-1} .
$$

Then it is easy to see that $\Psi \in B\left(\mathcal{H}, \sum_{i \in I} \bigoplus W_{i}\right)$ and $T_{W} \Psi=0$. However, the converse is not true. Indeed, consider $W_{1}=\operatorname{span}\{(1,1,0)\}$ and $W_{2}=\{0\} \times \mathbb{R}^{2}$, then $W=\left\{W_{i}\right\}_{i=1}^{2}$ is a 1-uniform fusion frame for $\mathbb{R}^{3}$. Now let $T_{W} \Psi=0$, then for every $f=(a, b, c) \in \mathbb{R}^{3}$ we have $\Psi^{*} T_{W}^{*} f=0$ so $\Psi^{*}\{((a+b) / 2,(a+b) / 2,0),(0, b, c)\}=0$. This implies that $\Psi^{*}\left\{f_{1}, f_{2}\right\}=0$, for every $\left\{f_{1}, f_{2}\right\} \in \sum_{i=1}^{2} \bigoplus W_{i}$. Hence the only 
operator $\Psi \in B\left(\mathcal{H}, \sum_{i=1}^{2} \oplus W_{i}\right)$ such that $T_{W} \Psi=0$ is $\Psi=0$. While, there exist more than one dual for $W$, such as $V=\left\{S_{W}^{-1} W_{1} \oplus \operatorname{span}\{(0,1 / 2,1)\}, S_{W}^{-1} W_{2}\right\}$ and $Z=\left\{S_{W}^{-1} W_{1}, S_{W}^{-1} W_{2} \oplus \operatorname{span}\{(1,0,3 / 2)\}\right\}$, which are 1-uniform alternate duals of $W$. Hence, unlike discrete frames, the corresponding assertion in Proposition 1.1 for fusion frames fails. This also shows that there is no one-to-one correspondence between duals of a fusion frame and bounded left inverses of $T_{W}^{*}$. However, we show that for the mapping $\Psi \in B\left(H, \sum_{i \in I} \bigoplus W_{i}\right)$ such that $T_{W} \Psi=0$ we can construct a dual for $F=\left\{\omega_{i} f_{i, j}\right\}_{i \in I, j \in J_{i}}$, where $\left\{f_{i, j}\right\}_{j \in J_{i}}$ is a local frame for $W_{i}$ for each $i \in I$. Define $\psi_{F}: \mathcal{H} \rightarrow l^{2}$ by $\psi_{F} f=\left\{c_{i, j}\right\}_{i \in I, j \in J_{i}}$ where $\{\Psi f\}_{i \in I}=\left\{\sum_{j \in J_{i}} c_{i, j} f_{i, j}\right\}_{i \in I}$ and $\left\{c_{i, j}\right\}_{j \in J_{i}}$ is a unique sequence whose elements represent the components of $\Psi f$ in $W_{i}$ with minimal $l^{2}$-norm, see Lemma 5.3.6 of [11]. The mapping $\psi_{F}$ is well defined since $\left\{c_{i, j}\right\}_{j \in J_{i}}$ is unique. Thus

$$
T_{F} \psi_{F} f=\sum_{i \in I, j \in J_{i}} \omega_{i} c_{i, j} f_{i, j}=\sum_{i \in I} \omega_{i}(\Psi f)_{i}=T_{W} \Psi f=0 .
$$

Therefore, we obtain a dual frame $\left\{g_{i, j}\right\}_{i \in I, j \in J_{i}}$ for $\left\{\omega_{i} f_{i, j}\right\}_{i \in I, j \in J_{i}}$ by Proposition 1.1. Hence, characterization of all dual fusion frames is an intricate problem.

3.2. Characterization of duals of some fusion frames. In the following, we try to characterize duals of some fusion frames in Hilbert spaces. First, we need to the following lemma.

3.5. Lemma. [9] Let $W_{1}$ and $W_{2}$ be closed subspaces of $\mathcal{H}$, and $\omega_{1}, \omega_{2}>0$. The following conditions are equivalent;

(i) $W=\left\{\left(W_{i}, \omega_{i}\right)\right\}_{i=1}^{2}$ is a Parseval fusion frame for $\mathcal{H}$.

(ii) Either $W_{1}=W_{2}=\mathcal{H}$ and $\omega_{1}^{2}+\omega_{2}^{2}=1$ or $W_{1} \perp W_{2}$ and $\omega_{1}=\omega_{2}=1$

3.6. Theorem. Let $W=\left\{\left(W_{i}, \omega_{i}\right)\right\}_{i=1}^{2}$ be a Parseval fusion frame and $V=\left\{\left(V_{i}, v_{i}\right)\right\}_{i=1}^{2}$ be a dual of $W$. Then one of the following conditions are hold:

(i) $V_{1}=V_{2}=\mathcal{H}$ and $\omega_{1} v_{1}+\omega_{2} v_{2}=1$.

(ii) $V_{1} \perp V_{2}$ and $\omega_{1} v_{1}=\omega_{2} v_{2}=1$.

(iii) $V_{1} \supseteq W_{1}, V_{2} \supseteq W_{2}$ and $v_{1}=v_{2}=1$.

Proof. Since $W$ is a Parseval fusion frame, therefore by Lemma 3.5 either we have $W_{1}=$ $W_{2}=\mathcal{H}$ and $\omega_{1}^{2}+\omega_{2}^{2}=1$ or we have $W_{1} \perp W_{2}$ and $\omega_{1}=\omega_{2}=1$. Now if $W_{1}=W_{2}=\mathcal{H}$, and $\omega_{1}^{2}+\omega_{2}^{2}=1$, then easily we can see that $\left(\left\{V_{i}, \sqrt{\omega_{i} v_{i}}\right\}_{i=1}^{2}\right)$ is a Parseval fusion frame and so we have either $(i)$ or $(i i)$. On the other hand, let $W_{1} \perp W_{2}$, and $\omega_{1}=\omega_{2}=1$. In this case, for every $f \in W_{1}$ we have $v_{1} \pi_{V_{1}} f=f$ and so $W_{1} \subseteq V_{1}$ and $v_{1}=1$, similarly $W_{2} \subseteq V_{2}$ and $v_{2}=1$, i.e., $(i i i)$ holds.

3.7. Theorem. Suppose that $W=\left\{\left(W_{i}, \omega_{i}\right)\right\}_{i=1}^{\infty}$ is a fusion frame for $\mathcal{H}$ such that $W_{1} \subseteq W_{2} \subseteq W_{3} \subseteq \ldots$, then a Bessel fusion sequence $V=\left\{\left(V_{i}, \omega_{i}\right)\right\}_{i=1}^{\infty}$ is a dual of $W$ if and only if $V_{i} \supseteq S_{W}^{-1} W_{i}$, for all $i$.

Proof. Let $V=\left\{\left(V_{i}, \omega_{i}\right)\right\}_{i=1}^{\infty}$ be a dual of $W$. If $S_{W}^{-1} W_{1}$ is not a subspace of $V_{1}$, then there exists $f_{0} \in W_{1}$ such that $S_{W}^{-1} f_{0} \in S_{W}^{-1} W_{1} \backslash V_{1}$ and so $\left\|\pi_{V_{1}} S_{W}^{-1} f_{0}\right\|<\left\|S_{W}^{-1} f_{0}\right\|$. Also

$$
\sum_{i=1}^{\infty} \omega_{i}^{2} \pi_{V_{i}} S_{W}^{-1} \pi_{W_{i}} f_{0}=f_{0}=\sum_{i=1}^{\infty} \omega_{i}^{2} S_{W}^{-1} \pi_{W_{i}} f_{0}
$$

Therefore,

$$
\sum_{i=1}^{\infty} \omega_{i}^{2}\left\|\pi_{V_{i}} S_{W}^{-1} f_{0}\right\|^{2}=\sum_{i=1}^{\infty} \omega_{i}^{2}\left\|S_{W}^{-1} f_{0}\right\|^{2}
$$


which is a contradiction. Now let $S_{W}^{-1} W_{1} \subseteq V_{1}, \ldots, S_{W}^{-1} W_{j-1} \subseteq V_{j-1}$ we show that $S_{W}^{-1} W_{j} \subseteq V_{j}$. Otherwise, as the above there exists an element $f_{0} \in W_{j}$ such that $S_{W}^{-1} f_{0} \in S_{W}^{-1} W_{j} \backslash V_{j}$. So we can write

$$
\sum_{i=j}^{\infty} \omega_{i}^{2} \pi_{V_{i}} S_{W}^{-1} f_{0}=\sum_{i=j}^{\infty} \omega_{i}^{2} S_{W}^{-1} f_{0} .
$$

Hence $\sum_{i=1}^{\infty} \omega_{i}^{2}\left\|\pi_{V_{i}} S_{W}^{-1} f_{0}\right\|^{2}=\sum_{i=1}^{\infty} \omega_{i}^{2}\left\|S_{W}^{-1} f_{0}\right\|^{2}$ which is a contradiction, therefore $S_{W}^{-1} W_{i} \subseteq V_{i}$ for all $i$.

It is worth noticing that, if $W=\left\{\left(W_{i}, \omega_{i}\right)\right\}_{i=1}^{n}$ is a fusion frame for $\mathcal{H}$ such that $W_{1} \supseteq W_{2} \supseteq \ldots \supseteq W_{n}$, then similar to the proof of Theorem 3.7 we can see that a Bessel fusion sequence $V=\left\{\left(V_{i}, \omega_{i}\right)\right\}_{i=1}^{n}$ is a dual of $W$ if and only if $V_{i} \supseteq S_{W}^{-1} W_{i}$, for all $1 \leq i \leq n$. Also, this condition that the weights of dual $V$ is equal to weights of fusion frame $W$ is necessary. For example, consider $\mathcal{H}=\mathbb{C}^{3}, W_{i}=\mathbb{C}^{3}$ and $\omega_{i}=1 / \sqrt{3}$, for each $1 \leq i \leq 3$. Clearly $W=\left\{\left(W_{i}, \omega_{i}\right)\right\}_{i=1}^{3}$ is a Parseval fusion frame with a dual as $V=\left\{\left(V_{i}, \nu_{i}\right)\right\}_{i=1}^{3}$, where $V_{1}=\mathbb{C}^{3}$ and $V_{2}=V_{3}=\{0\}$ and $v_{i}=\sqrt{3}$ for each $1 \leq i \leq 3$.

\section{References}

[1] Ali, S. T. Antoine, J. P. and Gazeau, J. P. Continuous frames in Hilbert spaces. Ann. Physics. 222, 1-37, 1993.

[2] Arefijamaal, A. and Zekaee, E. Signal processing by alternate dual Gabor frames, Appl. Comput. Harmon. Anal. 35, 535-540, 2013.

[3] Benedetto,J. Powell, A. and Yilmaz, O. Sigm-Delta quantization and finite frames. IEEE Trans. Inform. Theory. 52, 1990-2005, 2006.

[4] Bodmannand,B. G. and Paulsen, V. I. Frames, graphs anderasures, Linear. Algebra. Appl. 404, 118-146, 2005.

[5] Bolcskel, H., Hlawatsch, F. and Feichtinger, H. G. Frame-theoretic analysis of oversampled filter banks. IEEE Trans. Signal Process. 46, 3256-3268, 1998.

[6] Candes, E. J. and Donoho, D. L. New tight frames of curvelets and optimal representations of objects with piecewise $C^{2}$ singularities. Commun. Pure Appl. Anal. 56, 216-266, 2004.

[7] Casazza, P. G. and Kutyniok, G. Frames of subspaces, Contemp. Math. 345, 87-114, 2004.

[8] Casazza, P. G., Kutyniok, G. and Li, S. Fusion frames and distributed processing, Appl. Comput. Harmon. Anal. 25 (1), 114-132, 2008.

[9] Casazza, P. G., Kutyniok, G., Li, S. and Rozell, c. J. Modeling Sensor Networks with Fusion Frames, Wavelets XII (San Diego, CA, 2007), 67011M-1-67011M-11, SPIE Proc. 6701, SPIE, Bellingham, WA.

[10] Casazza, P. G., Kutyniok, G. and Lammers, M. C. Duality principles in frame theory, J. Fourier Anal. Appl. 10(4), 383-408, 2004.

[11] Christensen, O. Frames and Bases: An Introductory Course, Birkhäuser, Boston. 2008.

[12] Duffin, R. and Schaeffer, A. A class of nonharmonic Fourier series, Trans. Amer. Math. Soc. 72, 341-366, 1952.

[13] Găvruţa, P. On the duality of fusion frames, J. Math. Anal. Appl. 333 (2), 871-879, 2007.

[14] Kaftal, V., Larson, D. R. and Zhang, Sh. Operator-valued frames, Trans. Amer. Math. Soc. 361, 6349-6385, 2009.

[15] Leng, J., Gue, Q. and Huang, T. The duals of fusion frames for experimental data transmission coding of energy physics, Hindawi. Publ. Corp. 2013, 1-9, 2013.

[16] Mller, V. Spectral theory of linear operators and spectral systems in Banach algebras, Operator Theory: Advances and Applications, 139. Birkhuser Verlag, Basel, 2003.

[17] Ruiz, M. A. and Stojanoff, D. Some properties of frames of subspaces obtained by operator theory methods, J. Math. Anal. Appl. 343, 366-378, 2008.

[18] Sadeghi, Gh. and Arefijamaal, A. von Neumann-Schatten frames, Medi. J. Math. 9 (3), 525-535, 2012. 
[19] Sun, W. G-frames and G-Riesz bases. J. Math. Anal. Appl. 322, 437-452, 2006. 